\title{
LA LATERALIZACIÓN CEREBRAL Y EL ORIGEN DEL LENGUAJE
}

\author{
ANTONIO BENÍTEZ-BURRACO \\ (Universidad de Oviedo) \\ abenitez@us.es
}

\begin{abstract}
Resumen
El "órgano del lenguaje" se encuentra lateralizado en términos estructurales y funcionales. Se ha prestado una especial atención a aquellas evidencias fósiles que parecen sugerir la existencia en especies de homínidos extintas de una lateralización cerebral de carácter estructural y funcional semejante a la que se advierte en los humanos, que podrían ser un indicio de la disponibilidad de una (cierta) capacidad de procesamiento lingüístico. Evidencias adicionales han aportado nuevos datos sobre los principales hitos que habrían jalonado dicho itinerario evolutivo, en particular, en lo que concierne a la cuestión de la lateralización y su relación con el lenguaje. El objetivo de este trabajo es tratar de valorar críticamente todas estas evidencias, así como las inferencias realizadas a partir de ellas sobre las capacidades lingüísticas de otras especies de homínidos. La conclusión fundamental será que, mientras que las evidencias fósiles parecen enfrentarse a dificultades inherentes a la asunción de una homología funcional, alli donde parece existir (sólo) una homología estructural, las evidencias de naturaleza molecular parecen asumir una concepción demasiado simplista de las relaciones que se establecen entre los genes y la cognición. E artículo concluirá bosquejando un modelo evolutivo general del "órgano del lenguaje" más ajustado al creciente corpus de datos genéticos y moleculares.

PALABRAS CLAVE: lateralización cerebral, evidencias fósiles, biología molecular, evolución del lenguaje
\end{abstract}

\section{Abstract}

The "language organ" seems to be lateralised both structurally and functionally. When trying to discern how language evolved, fossil evidences concerning structural and functional brain lateralization have been regularly considered, and a (rudimentary) language faculty have tentatively been inferred for some extinct hominid species. Further evidences, mainly concerning brain gene evolution, have also been recently taken into account when trying to determine phylogenetic relationships between brain lateralization and language. This growing body of evidences are critically examined in this paper. A main conclusion will be that fossil evidences often lead to wrongly infer functional homology from structural homology, whereas molecular data are frequently linked to simplistic conceptions of the genuine connection between genes and cognition. Consequently, a tentative model for accounting the evolution of the language organ will be outlined, mainly based on genetic and molecular data.

KEYWORDS: brain lateralization, fossil evidences, molecular biology, language evolution 


\section{Introducción}

Cualquier inferencia legítima acerca del itinerario evolutivo seguido por un determinado rasgo biológico debe partir necesariamente de la comparación, en términos anatómicos y funcionales, de dicho rasgo con otros homólogos, existentes en especies vivas relacionadas con aquella que se analiza, o bien, del análisis de evidencias fósiles de etapas intermedias de dicho itinerario (Gould, 1991) ${ }^{1}$. Sin embargo, en el caso del lenguaje la primera posibilidad analítica parece resultar inviable, por cuanto se han extinguido las especies de homínidos que podrían haber poseído un sistema comunicativo semejante al nuestro, mientras que las especies de primates más cercanas a la humana en términos filogenéticos carecen de una facultad equivalente, ya que ninguna de ellas presenta, en particular, una herramienta de comunicación de naturaleza combinatoria que utilice simultáneamente como elementos combinables unidades que posean un valor simbólico (Botha, 2002). En lo que atañe a la segunda posibilidad, resulta evidente que ni el lenguaje, ni las principales estructuras biológicas relacionadas con el mismo (circuitos neuronales, partes blandas del aparato fonador, etc.) fosilizan, si bien se ha recurrido convencionalmente al análisis de determinadas evidencias secundarias presentes en el registro fósil para tratar de realizar inferencias acerca de la posesión por parte de determinadas especies de homínidos de una facultad lingüística equivalente a la humana, o cuando menos, para intentar arrojar luz sobre los sucesivos hitos que habría atravesado el lenguaje en su evolución. Algunas evidencias de este tipo son las que conciernen, en particular, a la existencia de una lateralización cerebral en términos estructurales y funcionales. El presente artículo, organizado como una sucesión de preguntas y respuestas necesariamente relacionadas, tiene por objeto tratar de dilucidar el genuino alcance que el análisis de la lateralización cerebral posee en lo concerniente al origen del lenguaje, prestando una especial atención a los siguientes aspectos de esta cuestión: (i) en qué sentido (y en qué medida) la lateralización puede considerarse un rasgo inherente al lenguaje humano; (ii) qué tipo de evidencias fósiles se ha venido considerando a la hora de tratar de demostrar la existencia de una lateralización estructural y funcional en especies extintas de homínidos; (iii) qué asumen realmente estas evidencias fósiles de la lateralización y hasta qué punto pueden considerarse concluyentes; (iv) qué otro tipo de evidencias (eminentemente moleculares) podrían utilizarse en este sentido y qué nuevas hipótesis se han planteado a partir de ellas; y (v) hasta qué punto pueden considerarse válidas ( $\mathrm{y}$ concluyentes) las inferencias que sobre el origen del lenguaje se han realizado a partir del análisis de todo este cúmulo de datos paleoantropológicos y moleculares.

\section{2. ¿Qué significa exactamente que el lenguaje “está lateralizado”?}

En líneas generales, la organización funcional del sistema nervioso central se sustenta en unos principios relativamente sencillos, que incluyen (i) la existencia de una interrelación

\footnotetext{
${ }^{1}$ Este trabajo ha sido realizado al amparo del proyecto de investigación "Biolingüística: fundamento genético, desarrollo y evolución del lenguaje" (HUM2007-60427/FILO), subvencionado por el Ministerio de Educación y Ciencia, con financiación parcial FEDER.
} 
funcional entre las diferentes regiones mediante el establecimiento de un patrón específico de interconectividad intrahemisférica e interhemisférica; (ii) el diseño de un sistema centralizado de procesamiento de los estímulos y de generación de las respuestas correspondientes; (iii) el establecimiento de una jerarquía en la organización neuroaxial (de manera que la complejidad funcional se incrementa conforme aumenta la cefalización); (iv) la aparición de una especialización funcional a nivel histológico; (v) la presencia de una organización topográfica de las vías neuronales y de sus proyecciones; (vi) la existencia de una plasticidad neuronal, con importantes repercusiones en lo concerniente a la posibilidad de modificar la función de determinadas neuronas y la organización de circuitos neuronales concretos; y, finalmente, (vii) la existencia de una lateralización, que implica una especialización funcional diferencial de los circuitos neuronales existentes en ambos hemisferios, la cual conlleva (y suele basarse en) un desarrollo diferencial concomitante de las estructuras neuronales (Hernández-Muela et al., 2004).

Si bien es cierto que los diferentes modelos neuronales de procesamiento lingüístico difieren en cuanto a la identidad precisa de las regiones del córtex cerebral (y aun de las subcorticales) que participan en el proceso, así como en lo que atañe al modo exacto en que se produce la interconexión entre las mismas, lo cierto es que casi todos coinciden en señalar que la mayor parte de las estructuras neuronales implicadas en el procesamiento de estímulos lingüísticos se hallan radicadas en el hemisferio cerebral izquierdo (para una revisión, cfr. Martin, 2003). Regiones particularmente significativas a este respecto (con independencia de que sean capaces, asimismo, de procesar otro tipo de estímulos) son, desde luego, el área de Broca, situada en el lóbulo frontal inferior; el área de Wernicke, localizada en la confluencia de los lóbulos parietal y temporal; y la circunvolución angular (Neville y Bavelier, 1998). Ahora bien, los análisis mediante técnicas de imagen no invasivas de la actividad cerebral in vivo durante la realización de tareas de índole lingüística han permitido constatar que conforme se incrementa la complejidad del mensaje que es preciso codificar/decodificar se produce un reclutamiento progresivo de otras regiones corticales, fundamentalmente como consecuencia de un incremento de la demanda computacional por parte de la memoria de trabajo verbal. Sin embargo, se ha constatado también que el hemisferio cerebral derecho (fundamentalmente las regiones homólogas a las áreas de Broca y Wernicke [Just et al., 1996]) sólo parece intervenir en aquellas tareas de procesamiento que superan los límites de la oración, como es el caso del análisis prosódico (Friederici, 2002) o del procesamiento del discurso (St George et al., 1999). Así, la presencia, en particular, de lesiones en el hemisferio derecho se ha correlacionado con una menor capacidad para procesar aspectos convencionalizados del discurso, como sucede, por ejemplo, con las metáforas o los modismos (Van Lancker y Kempler, 1987; Brownell et al., 1997), si bien en estos casos no parece verse afectada la capacidad de procesamiento sintáctico per se (Grodzinsky, 2000). En cierta medida algunas regiones del hemisferio derecho se han relacionado también con la organización de la información semántica (Mummery et al., 2000).

Por otro lado, merece la pena reseñar que esta "lateralización del lenguaje" está presente, igualmente, desde el momento del nacimiento (Friederici, 2006), lo que sugiere que existe una notable continuidad a este respecto a lo largo del desarrollo (en términos 
estructurales, pero también funcionales), en particular, en lo concerniente a las áreas involucradas habitualmente en el procesamiento lingüístico. Así, por ejemplo, están ya presentes antes de nacer las principales asimetrías estructurales, como es el caso del mayor tamaño del plano temporal del hemisferio izquierdo o de la menor extensión de la cisura de Silvio del hemisferio derecho (Witelson y Pallie, 1973). Del mismo modo, desde el momento del nacimiento se observa también una activación preferente de estas áreas en respuesta a los estímulos de naturaleza sonora, especialmente en el caso del plano temporal (Dehaene-Lambertz, et al., 2002), aunque es cierto que en una primera instancia esta activación asimétrica no parece depender de la naturaleza específicamente lingüística del estímulo. Sea como fuere, resulta preciso consignar, asimismo, que esta precoz organización lateralizada posee una significativa flexibilidad, especialmente durante los primeros años de vida, de modo que en determinados casos en los que se han producido importantes daños cerebrales durante las primeras fases del desarrollo puede producirse una "transferencia del lenguaje" al hemisferio derecho, merced a la plasticidad neuronal inherente a las estructuras implicadas en el procesamiento lingüístico (Thomas et al., 1997).

\section{3. ¿En qué sentido esta cuestión de la lateralización resulta relevante a la hora de discutir el origen del lenguaje?}

Tradicionalmente las diferentes hipótesis que se han venido planteando acerca del origen del lenguaje se han basado, en buena medida, en evidencias aportadas por la propia Lingüística. Sin embargo, en último término el lenguaje es, como se ha apuntado al responder la cuestión anterior, el resultado de la actividad de un conjunto interconectado de estructuras neuronales, el cual posee, para Anderson y Lightfoot (2000), la categoría de órgano, en el sentido de que constituiría una estructura especializada diseñada para satisfacer una función determinada, si bien, y en atención también a lo discutido anteriormente, este órgano no tendría una localización anatómica fácilmente discernible. Plantearse cuál es el origen del lenguaje supone, por consiguiente, tratar de establecer la procedencia evolutiva de este "órgano lingüístico". Así pues, y de igual modo que se ha venido haciendo en el caso de otros órganos corporales y de otras especies, existe una línea de investigación que se interesa por las evidencias fósiles del origen del lenguaje (para una revisión, cfr. Benítez-Burraco, 2003). Ahora bien, dado que el propio lenguaje no fosiliza, dichas evidencias se relacionan fundamentalmente con (i) la evolución estructural y funcional del cerebro, (ii) la evolución del aparato fonador y las estructuras del habla, y (iii) la aparición del pensamiento simbólico. Dentro de las evidencias de tipo paleoneurológico, que es como suele conocerse a las que integran el primer grupo, las más relevantes serían (a) las que constituyen un indicio de la ocurrencia de un incremento del volumen cerebral, el cual habría dado lugar a un aumento de la complejidad del cerebro en términos estructurales y funcionales, sentando las bases para el desarrollo de habilidades cognitivas complejas, como es el caso del lenguaje; en relación con esta cuestión, Chomsky ha sugerido que este aumento del volumen cerebral habría originado específicamente la reconfiguración de determinados circuitos neuronales necesaria para la aparición de una capacidad de combinación ilimitada (recursiva) de lo que en un principio habrían sido 
primitivos conceptuales, es decir, para la emergencia de lo que él denomina "facultad del lenguaje en sentido estricto" (FLE) (Hauser et al., 2002); (b) las que sugieren la aparición de las estructuras neuronales que en nuestra especie se encargan del procesamiento del lenguaje; y (c) las que constituyen, precisamente, una prueba de que dichas estructuras (y, por consiguiente, el "órgano del lenguaje") tienen un carácter lateralizado en términos anatómicos $\mathrm{y}$, sobre todo, funcionales.

\section{4. ¿Qué tipos de evidencias fósiles parecen sugerir la existencia de un cerebro lateralizado?}

Las evidencias fósiles que parecen indicar la existencia de una lateralización cerebral pueden agruparse en dos categorías diferentes. Por un lado se encuentran aquellas que sugieren la presencia de una lateralización estructural. Entre ellas las más significativas conciernen a la existencia de un desarrollo diferencial de las áreas que en nuestra especie intervienen en el procesamiento del lenguaje. Este tipo de análisis suele llevarse a cabo mediante el examen de las impresiones cerebrales fósiles. El estudio del endocráneo, es decir, de la superficie interna de los huesos del cráneo, permite determinar con cierto detalle la morfología externa del encéfalo, y en particular, la presencia de surcos, circunvoluciones, cisuras y venas meníngeas (Arsuaga et al., 2000). La principal conclusión de este tipo de análisis es que la morfología cerebral moderna se encuentra ya presente en el Homo habilis, que vivió hace entre 1,6 y 2,3 millones de años, y que sería el primer homínido dotado de las bases neuroanatómicas necesarias para el lenguaje (Wilkins y Wakefield, 1995). Así, diversos autores creen posible identificar, por ejemplo, un área de Broca con un desarrollo semejante al que existe en el ser humano en los restos fósiles de esta especie (Holloway, 1983; Tobias, 1983), la cual, según otros investigadores, podría estar presente incluso en el Australophitecus africanus, que vivió hace entre 2,4 y 3,5 millones de años (Tobias, 2003), en el que, por consiguiente, también se habría producido ya un descenso del surco parietoccipital, que se ha asociado a la ocurrencia de una reorganización de la zona cerebral posterior, la cual estaría relacionada a su vez con el surgimiento de los mecanismos asociativos multimodales necesarios para la representación de los conceptos lingüísticos (Holloway, 1983); no obstante, este tipo de remodelación parece más evidente en el caso del Homo habilis (Wilkins y Wakefield, 1995).

En lo que se refiere a la lateralización funcional, las evidencias más significativas atañen a la presencia de señales indicativas de un uso preferente de la mano derecha en la manipulación de objetos, que aparecen documentadas ya desde el Homo ergaster (Arsuaga et al., 2000). A diferencia de lo que sucede en otras especies de primates, la prevalencia del uso de la mano derecha en nuestra especie parece oscilar en torno al $90 \%$ desde hace, cuando menos, 5000 años, según se desprende del análisis de productos manufacturados que proceden de registros de épocas ya históricas (Coren y Porac, 1977). 


\section{5. ¿Por qué un cerebro (fósil) lateralizado (en términos motores) debería ser también un cerebro lingüístico (toda vez que uno lingüístico sí parece estar lateralizado)?}

La legitimidad que reclaman para sí las evidencias fósiles relacionadas con la lateralización estructural del cerebro resulta, en principio, fácil de aceptar, por cuanto dichas evidencias hacen referencia, en último término, a la posibilidad de que exista una correlación entre determinadas estructuras neuronales y determinadas funciones lingüísticas ( $o$, en general, entre diversas regiones cerebrales y el lenguaje como un todo), una propuesta que viene siendo habitual en la mayor parte de los modelos neuronales de procesamiento lingüístico correspondientes a nuestra especie. Sin embargo, no cabe decir lo mismo en lo que atañe a las evidencias relacionadas con la lateralización funcional. Antes bien, resultaría razonable plantearse en virtud de qué tipo de indicios cabe concluir que los efectos de lo que parece ser una lateralización motora impliquen también la existencia del lenguaje, aun cuando el procesamiento lingüístico de halle efectivamente lateralizado (también en términos funcionales) en nuestra especie. El principal cúmulo de evidencias a este respecto concierne a la correlación positiva que parece existir en el ser humano entre la habilidad verbal general y la precocidad del desarrollo del lenguaje, por un lado, y el grado de lateralización en el manejo de la mano, por otro, y, en general, entre la lateralización y las habilidades cognitivas, incluyendo el lenguaje (Leask y Crow, 2001), aunque lo cierto es que este efecto, aunque real, no es excesivamente significativo, puesto que tan sólo sería responsable de una variación de alrededor del $1 \%$ en el índice de Habilidad General y en el coeficiente intelectual, y de un porcentaje semejante en lo que atañe al componente verbal del mismo (Nettle, 2003). No obstante, este tipo de correlaciones posee un mayor calado, en lo que atañe a la evolución de los mecanismos neuronales implicados en el procesamiento del lenguaje, que el que parecen cuando se contemplan en términos meramente cuantitativos, a la luz fundamentalmente de determinadas propuestas que sugieren que el sustrato neuronal del lenguaje, en la especie humana, y de determinadas tareas motoras, en otras especies de primates, podrían compartir un origen común en términos filogenéticos (vid infra).

\section{6. ¿Qué tipo de problemas presentan este tipo de evidencias fósiles?}

En lo que concierne específicamente a las evidencias fósiles que sugerirían la existencia de una lateralización estructural, lo cierto es que el análisis de los endocráneos únicamente proporciona información de tipo anatómico acerca de la porción superficial del córtex cerebral, de manera que se ha sugerido que resultaría metodológicamente cuestionable la realización de inferencias acerca de la organización neuroanatómica global del cerebro (y, desde luego, de su organización funcional) a partir de este tipo de evidencias. Un caso particularmente ilustrativo a este respecto lo representan las estructuras subcorticales, que desempeñan un papel fundamental en determinados modelos de procesamiento lingüístico, como es el caso del Sistema Funcional del Lenguaje (Lieberman, 2002), donde se encargarían del procesamiento de las tareas secuenciales asociadas a la fonación y la sintaxis. Por lo demás, y como se sugirió en la primera de las cuestiones, las funciones 
cognitivas complejas no parecen corresponderse de forma tan unívoca como se venía creyendo habitualmente con las estructuras neuroanatómicas (cfr. también Dingwall, 1995; Donald, 1995 y Whitcombe, 1995 a este respecto), de forma que una descripción exhaustiva de los centros lingüísticos (también en nuestros antepasados más inmediatos) debería contemplar la consideración de otras regiones, adicionales a la del córtex perisilviano del hemisferio izquierdo (que son las analizadas habitualmente), debido al reclutamiento recurrente que durante el procesamiento lingüístico se hace de regiones cerebrales que se consideraban encargadas, en principio, de tareas no lingüísticas.

En lo que atañe a las evidencias concernientes a la lateralización funcional y a su relación con el lenguaje, resulta evidente que la relevancia que puede revestir la existencia de una correlación positiva entre ambos parámetros queda sustancialmente relativizada por la reducida contribución cuantitativa de la primera al componente verbal de la actividad cognitiva, tal como se cuantificó anteriormente.

\section{7. ¿Existen otras evidencias al margen de las de tipo fósil capaces de atestiguar la existencia de la lateralización?}

Más allá del estudio del registro fósil, un modo alternativo de dilucidar el proceso evolutivo que ha dado lugar a la emergencia de las capacidades cognitivas privativas de la especie humana consiste en el análisis de las mutaciones acaecidas en algunos de los genes que integran el programa molecular que interviene en la regulación del desarrollo y del funcionamiento del sistema nervioso central: en general, de aquellas que han sido objeto de una selección positiva a lo largo de nuestra reciente historia evolutiva, y en particular, las experimentadas por aquellos genes cuya mutación en nuestra especie modifica características generales de organización y actividad cerebrales (volumen, lateralización funcional, modularidad anatómica y/o funcional) que serían relevantes para la aparición del lenguaje en términos filogenéticos y cuya evolución se ha querido determinar mediante el estudio de determinadas evidencias indirectas procedentes del registro fósil, tal como se discutió anteriormente. Allí donde el examen de los restos paleoneurológicos puede plantear determinadas incertidumbres acerca de su identificación o datación precisas (y, desde luego, de su significado biológico y sus implicaciones en lo concerniente a la evolución del lenguaje), las técnicas de las que hace uso la biología molecular, partiendo del análisis de las diferencias genéticas existentes entre las secuencias de los genes humanos y de los genes homólogos de los primates superiores, permiten datar cronológicamente, con particular exactitud, los cambios (genéticos) que se han ido produciendo a lo largo de nuestra especiación, llegando a proponer, en último término, posibles causas (moleculares) para las modificaciones neuroanatómicas y neurofuncionales que han tenido lugar durante la misma $\mathrm{y}$, en particular, para aquellas que, como ocurre con la lateralización estructural y funcional del cerebro, parecen revestir una cierta importancia en lo que atañe a la aparición del lenguaje. 


\section{Si estamos hablando de genes, es presumible que haya sido posible determinar la existencia de una base genética para la lateralización funcional del cerebro. ¿Es eso cierto?}

Se han llevado a cabo diversos análisis para tratar de establecer si la dominancia de la mano derecha y, por extensión, la dominancia y la asimetría cerebrales, tienen una base genética (McManus, 1985; Lalland et al., 1995; Yeo et al., 1997), si bien los resultados obtenidos hasta el momento son particularmente variables. Para algunos investigadores dicha dominancia dependería de un único gen (Klar, 1996); para otros, lo haría de dos genes con cuatro alelos (Levy y Nagylaki, 1972); para un tercer grupo, se trataría de un carácter multifactorial (Francks et al., 2002; Francks et al., 2003); para un cuarto grupo dependería de factores hormonales (Olsson e Ingvar, 1991); mientras que para un último grupo de investigadores sería exclusivamente una cuestión de transmisión cultural (Bishop, 2001). En todo caso, parece existir la certeza de que el control genético es más acusado en los casos en que los existe una dominancia de la mano derecha, de manera que el peso del ambiente sería más importante cuando domina la mano izquierda (Geschwind et al., 2002). El análisis llevado a cabo por Bishop (2001) resulta especialmente interesante en el contexto de este trabajo, puesto que su objetivo era tratar de determinar la existencia de una hipotética asociación entre la dominancia de una de las dos manos y los trastornos específicos del lenguaje y del habla, así como dilucidar si dicha asociación, en caso de existir, se debía al efecto de un mismo gen o de diferentes genes. Como se apuntó anteriormente, las conclusiones resultaron negativas en ambos casos. No obstante, recientemente se han realizado varios análisis de ligamiento para tratar de determinar la localización y, en su caso, la naturaleza, de los hipotéticos QTLs (loci de caracteres cuantitativos, cuya utilización busca fundamentalmente llevar a cabo una detección de múltiples genes con un efecto relativamente pequeño [Risch y Merikangas, 1996]) relacionados con este carácter, habiéndose identificado diversos picos de ligamiento, situados en cromosomas diferentes. El de mayor significación estadística estaría localizado en la región cromosómica 2p12-q11, mientras que existiría otro locus (o lugar físico en el cromosoma) adicional en 17p11-q23 (Francks et al., 2002; Francks et al., 2003). Otras regiones potencialmente interesantes a este respecto podrían estar situadas en los cromosomas 1 y 10, en particular, en las regiones cromosómicas que contienen, respectivamente, los genes DNAHC13 y NODAL (Van Agtmael et al., 2002); este último gen codifica un factor de crecimiento de tipo TGF- $\beta$ que interviene en el establecimiento de la simetría bilateral durante el desarrollo embrionario (Zhou et al., 1993; Lowe et al., 1996; Gebbia et al., 1997). No obstante, este último resultado está pendiente de replicación, puesto que ha sido descartado por otros análisis, en particular por el realizado por McManus (1985), quien trataba de determinar la relación existente entre la dominancia en el uso de una mano, las capacidades lingüísticas y la afasia. 


\section{9. ¿Qué hipótesis más significativas se han planteado a este respecto en relación con el origen del lenguaje?}

En lo concerniente a los fundamentos genéticos de la lateralización cerebral y al origen del lenguaje, la propuesta más llamativa es seguramente la de Crow (2001): en consonancia con la variación sexual encontrada en el grado de lateralización y de habilidad lingüística en el ser humano, Leask y Crow (2001) esperan que, cuando menos, uno de los genes implicados se encuentre en los cromosomas sexuales (Corballis et al., 1996) y que su expresión se halle sometida a una regulación epigenética. Para estos investigadores el candidato más idóneo sería el gen de la protocadherina 11 , del que existen, consecuentemente, dos variantes, una ligada a X (PCDHX $[P C D H 11 X])$, localizada en Xq21.3, y otra ligada a Y (PCDHY [PCDH11Y]), localizada en Yp11.2, pero cuya identidad alcanza el $98.3 \%$ a nivel amioacídico (Blanco et al., 2000). La expresión de ambos genes sería diferente en los dos sexos, puesto que el gen PCDHX $[P C D H 11 X]$ escapa, en particular, al proceso general de inactivación que afecta característicamente a los genes contenidos en el cromosoma X, de ahí que sus niveles de expresión en los individuos de sexo femenino dupliquen los observados en los de sexo masculino (Lopes et al., 2006). El gen $P C D H Y[P C D H 11 Y]$ es el resultado de un proceso de transposición al cromosoma Y de una copia del gen $P C D H X[P C D H 11 X]$, el cual tuvo lugar hace entre tres y cuatro millones de años, tras la separación de la línea evolutiva que condujo a la aparición de la especie humana de la que dio lugar a las restantes especies actuales de primates (Sargent et al., 1996), de ahí que esté ausente en dichas especies (Wilson et al., 2006) y que no se observe en ellas la disparidad sexual en el grado de lateralización, ni consecuentemente de habilidad manual, que aparece característicamente en nuestra especie. En todo caso, lo relevante en el contexto de este artículo es el hecho de que la datación del evento de reorganización cromosómica se aproxima en buena medida a la datación sugerida por las evidencias fósiles de la lateralización funcional a las que se hizo referencia anteriormente (los primeros restos del Homo ergaster tienen una antigüedad de alrededor de dos millones de años [Arsuaga et al., 2000]).

Conviene tener presente que la superfamilia de las cadherinas, a la que pertenecen este tipo de protocadherinas, incluye un amplio grupo de proteínas implicadas en la adhesión celular homofílica. En el sistema nervioso central las cadherinas regulan la diferenciación de las poblaciones neuronales y el desarrollo de estructuras cerebrales, proporcionando un código específico a los distintos subgrupos funcionales de neuronas; asimismo, están implicadas en la regulación del crecimiento de los axones y en la modulación de las características estructurales y funcionales del espacio sináptico, mediante su anclaje al citoesqueleto celular (Shapiro y Colman, 1999; Bruses, 2000; Benson et al. 2001). Resulta muy interesante el hecho de que la propia actividad neuronal puede regular la expresión de los genes que codifican estas proteínas, como sucede, por ejemplo, en el caso de la protocadherina arcadlina (Yamagata et al., 1999). El atractivo adicional que presentan específicamente las protocadherinas se debe al hecho de que se trata de miembros de la superfamilia particularmente recientes desde el punto de vista evolutivo, que además de mediar la adhesión intercelular homofílica, también parecen participar en interacciones 
heterofílicas, pudiendo comportarse adicionalmente como moléculas receptoras o señalizadoras (Frank y Kemler, 2002); de hecho, las protocadherinas interaccionan con diversos componentes citoplasmáticos implicados en la regulación de la plasticidad sináptica y de la organización citológica del córtex cerebral (Frank y Kemler, 2002).

\section{0. ¿Qué objeciones cabría hacer específicamente a este tipo de propuestas?}

Una propuesta como la anterior resulta paradigmática en relación con la tendencia que en ocasiones puede observarse a simplificar desde el punto de vista genético lo que desde el campo de la neurociencia cognitiva o la psicolingüística se viene describiendo como procesos extremadamente complejos. Resulta preciso subrayar en este sentido que los genes no codifican funciones, sino determinados productos bioquímicos (la mayoría de naturaleza proteínica), los cuales actúan de forma coordinada junto con los productos codificados por otros muchos genes, en un determinado lugar del cerebro y en un momento concreto del desarrollo, para permitir determinados procesos celulares; por lo demás, el efecto que ejercen los productos codificados por los genes está condicionado en gran medida por el contexto molecular y ontogenético, así como por el ambiente, de modo que el componente molecular del lenguaje influye en (y se ve influido por) los restantes niveles de complejidad biológica (celular, fisiológico, funcional, macroestructural y fenotípico [lingüístico]) del "órgano del lenguaje" (Benítez-Burraco, 2007). Así pues, y en relación específicamente con la propuesta discutida en la cuestión precedente, no cabe sino relativizar el efecto que sobre la aparición del lenguaje habría tenido la modificación de este único gen (especialmente si tenemos en cuenta además la reducida contribución cuantitativa de la lateralización al componente verbal de la actividad cognitiva en la especie humana). Eso no quiere decir, no obstante, que la búsqueda de los "genes del lenguaje" (en el sentido en que los define Pinker [2001: 352-353], quien los ha caracterizado como "secuencias de ADN que codifican proteínas o desencadenan la trascripción de otras proteínas, en determinados momentos y lugares del cerebro, que guían, fijan o atraen neuronas hacia aquellos circuitos que, una vez producido el ajuste sináptico que tiene lugar con el aprendizaje, intervienen en la solución de problemas gramaticales") deba dejar de lado este tipo de genes, que, con toda probabilidad, desempeñan un papel muy importante en la conformación de los circuitos neuronales que intervienen en el procesamiento de estímulos lingüísticos. En todo caso, lo relevante en este empeño no sería tanto la identificación de genes implicados exclusivamente en el desarrollo y en el funcionamiento de dichos circuitos (aunque es posible que existan algunos), cuanto la disección de la arquitectura exacta del programa genético único que participa en la regulación de ambos procesos, el cual estaría integrado por genes que, en la inmensa mayoría de los casos, desempeñan funciones diferentes en lugares distintos del cerebro ( $\mathrm{y}$ aun en otras regiones corporales) y en las distintas etapas de la ontogenia del organismo. Y lo mismo podría decirse desde un punto de vista filogenético. 


\section{1. ¿Y, en general, a la correlación entre lateralización y lenguaje en términos filogenéticos?}

Como se discutió en la primera de las cuestiones recogidas en este trabajo, la lateralización del cerebro humano tiene un carácter anatómico y funcional. Sin embargo, la lateralización funcional es, en sí misma, una característica arcaica dentro de los vertebrados (Bradshaw y Nettleton, 1981). Del mismo modo, la lateralización anatómica de las regiones más significativas en relación con el procesamiento lingüístico, como sucede, en particular, con el área de Broca, constituye un rasgo compartido por el ser humano y por otros primates cuyas capacidades lingüísticas son claramente diferentes de las nuestras (Cantalupo y Hopkins, 2001), de manera que se ha estimado que la antigüedad del sustrato neuroanatómico para dicha dominancia puede retrotraerse a hace, cuando menos, cinco millones de años (Cantalupo y Hopkins, 2001). El problema estriba, sin embargo, en que el área cerebral homóloga al área de Broca humana contiene en los primates neuronas especulares implicadas en el control motor manual, las cuales no sólo se descargan durante la manipulación, como sería esperable, sino también durante la observación de la misma (Rizzolatti y Arbib, 1998). Aunque las implicaciones (proto)lingüísticas de este sistema son evidentes, desde el momento en que supondría la existencia de una paridad entre actor y espectador, al tener cualquier secuencia de hechos el mismo "significado" para ambos (Arbib, 2003), lo relevante en relación con la cuestión tratada en este trabajo es que la existencia de una homología en términos estructurales no implicaría que dicha homología se conservase, asimismo, desde el punto de vista funcional.

En atención a este tipo de evidencias, se ha sugerido, desde luego, que el área de Broca humana (en tanto que estructura neuronal dedicada al procesamiento del lenguaje) podría haber evolucionado a partir de una región encargada en nuestros antecesores de generar y reconocer las secuencias motoras que permiten la manipulación de los objetos (Rizzolatti et al., 1996), de ahí el interés adicional (y la legitimidad) que posee a priori el análisis de las evidencias fósiles de la lateralización funcional a las que se hizo referencia anteriormente. En relación con esta cuestión, conviene tener en cuenta, asimismo, que en determinados trastornos cognitivos en los que se advierte un déficit en el componente pragmático del lenguaje y/o en la capacidad de construir correctamente una "teoría de la mente" (el caso paradigmático sería el del autismo) se ha observado una correlación inversa entre la gravedad de los mismos y la actividad de este tipo de neuronas especulares, en concreto, de las que forman parte del giro frontal inferior (pars opercularis) (Dapretto et al., 2006), una región sobre la que existen numerosas evidencias (derivadas fundamentalmente de los análisis in vivo realizados mediante técnicas de imagen no invasivas) acerca de su implicación en el procesamiento lingüístico, en particular, en el de carácter sintáctico (Freedman y Martin, 2001; Ben-Shachar et al., 2003). Sin embargo, la mera consideración de este tipo de datos, aunque sea en conjunción con las evidencias que sugieren la existencia de una lateralización anatómica, resulta insuficiente para poder realizar inferencias legítimas acerca del momento en que una región como el área de Broca pudo comenzar a encargarse del procesamiento de estímulos lingüísticos. De ahí el interés que ha despertado, en la línea de lo apuntado al tratar la sexta de las cuestiones que integran este artículo, el análisis de la 
historia evolutiva de determinados genes cuya mutación en la especie humana origina alteraciones del lenguaje de diversa índole. En relación con esta cuestión, un caso particularmente relevante es el de FOXP2, cuya mutación da lugar a trastornos de diversa naturaleza y alcance, pero que son simultáneamente motores y lingüísticos, y que afectan tanto al componente receptivo del lenguaje como al expresivo, de modo que en los individuos que portan una copia defectuosa del gen se advierten (i) un menor nivel de conocimientos de tipo léxico; (ii) una menor capacidad de comprensión morfológica y morfosintáctica; (iii) problemas de generación morfológica, (iv) un déficit en la capacidad de almacenamiento de información fonológicamente relevante por parte de la memoria de trabajo verbal o quizás en el proceso de articulación secuencial de unidades con valor fonológico; (v) una menor capacidad de recuperación de elementos del lexicón (en términos cuantitativos y cualitativos); y (vi) una dispraxia orofacial ligada al desarrollo, o bien, una disartría espástica, que iría acompañada de una dispraxia verbal y de errores residuales durante el mismo (Gopnik y Crago, 1991; Vargha-Khadem et al., 1995; Watkins et al., 2002; Shriberg et al., 2006). El gen codifica un factor transcripcional que interviene en la regulación de la diferenciación neuronal necesaria para la organización y/o el funcionamiento de determinados circuitos córtico-talamo-estriatales asociados a la planificación motora, el comportamiento secuencial y el aprendizaje procedimental (para una revisión, cfr. Marcus y Fisher, 2003; Benítez-Burraco, 2005a; Vargha-Khadem et al., 2005). Dos mutaciones que han afectado presumiblemente a la función de la proteína codificada por este gen han sido seleccionadas coincidiendo con la aparición del Homo sapiens, hace alrededor de 200.000 años (Enard et al., 2002a) o quizás en el antepasado común al Homo sapiens y al Homo neanderthalensis, hace entre 300.000 y 400.000 años (Krause et al., 2007). En todo caso, y en atención fundamentalmente al descenso que se advierte en la actividad del área de Broca durante tareas de procesamiento lingüístico en los individuos que presentan una copia mutada del gen (cfr. a este respecto Liégeois et al., 2003), se ha sugerido que dichas mutaciones habrían permitido a la nueva proteína amplificar las funciones asociadas inicialmente a esta región cortical, facilitando, en particular, la emergencia de la sintaxis u optimizando el procesamiento fonológico y/o la memoria de trabajo verbal. Resulta también plausible, y en consonancia en este caso con la dispraxia orofacial observada en quienes presentan una variante disfuncional de la proteína FOXP2, que dichas mutaciones hubieran permitido, asimismo, reclutar al área de Broca para lograr un control voluntario y más preciso de los movimientos orofaciales, lo que resulta imprescindible para el desarrollo del dispositivo articulatorio del que hace uso la especie humana para la codificación y emisión de los mensajes lingüísticos (para una revisión sobre estas cuestiones, cfr. Benítez-Burraco, 2005b).

Los riesgos que plantea la asunción de la existencia de una homología funcional cuando se constata la presencia de una homología estructural quedan puestos de manifiesto, igualmente, por el caso del área de Wernicke. Así, los grandes simios presentan también un plano temporal izquierdo de mayor tamaño (esta región incluye el área de Wernicke), aunque es cierto que las diferencias citoarquitectónicas que se advierten habitualmente en el caso de esta estructura entre ambos hemisferios cerebrales son de menor entidad que las que existen en nuestra especie (Gannon et al., 1998; Cantalupo et al., 2003), como sucede 
paradigmáticamente en el caso del córtex temporal (Buxhoeveden et al., 2001). Por otro lado, en los macacos se observa una activación preferente del polo temporal del hemisferio izquierdo en respuesta a la audición de las vocalizaciones características de la especie (Poremba et al., 2004). Esta respuesta asimétrica parece estar ligada, además, a la prominencia comunicativa de las llamadas y no tanto a sus características acústicas, al igual que sucede en nuestra especie (Petersen et al., 1984). Finalmente, y como también ocurre en el ser humano, esta asimetría se desarrolla conforme aumenta la exposición a los estímulos en cuestión, de forma que está ausente en los individuos recién nacidos (Hauser y Andersson, 1994). Ahora bien, como es sabido, en la especie humana la región del plano temporal (y, en particular, el área de Wernicke) desempeña otras funciones adicionales, ligadas de modo mucho más directo al procesamiento lingüístico, por cuanto parece intervenir en la organización de la información de carácter semántico, constituyendo parte del sustrato neuronal del diccionario mental o lexicón (Damasio et al., 1996), aunque también parece ser un componente de la memoria de trabajo verbal (Lieberman, 2002).

Así pues, la disparidad que se advierte entre nuestra especie y las restantes especies de primates a nivel funcional entre regiones que, en términos anatómicos, presentan una organización semejante, especialmente en lo que concierne a su lateralización estructural (casos paradigmáticos serían los de las áreas de Broca y Wernicke discutidas anteriormente) parece sugerir que durante la evolución de la especie humana se habría producido una reutilización con fines lingüísticos de las áreas homólogas presentes en nuestros antepasados (Cantalupo y Hopkins, 2001), las cuales conservan probablemente una función semejante a la original en los primates, en los que su organización es también asimétrica y en los que se encargan de funciones no lingüísticas (MacNeilage, 1998). La existencia en estas áreas, en especies próximas al hombre, de neuronas especulares integradas únicamente en circuitos encargados del control motor manual parece corroborar la hipótesis de que en aquella transición podría haber existido una etapa intermedia en la que la transmisión de información se habría producido mediante gestos (Corballis, 2002), algo que, por otro lado, reforzaría el interés que posee el análisis de las evidencias fósiles de la lateralización funcional.

Conviene no olvidar, por último, que, tal como puede colegirse de lo discutido anteriormente, los análisis paleoneurológicos se han centrado habitualmente en la identificación y la caracterización de las áreas de Broca y Wernicke existentes presumiblemente en los endocráneos fosilizados, siguiendo en buena medida, la primitiva dicotomía anatómica establecida entre ambas regiones en lo que atañe al procesamiento del lenguaje, que implicaba una dicotomía funcional entre sintaxis y semántica. Ahora bien, teniendo presente, asimismo, lo discutido con anterioridad a este respecto, una descripción exhaustiva de los centros lingüísticos (también en nuestros antepasados más inmediatos) debería contemplar la consideración de otras regiones, adicionales a la del córtex perisilviano del hemisferio izquierdo, debido al reclutamiento recurrente que durante el procesamiento del lenguaje se hace de regiones cerebrales que se han venido considerando encargadas de tareas no lingüísticas, según se desprende de un corpus creciente de evidencias clínicas y de resultados procedentes de los estudios de neuroimagen (para una revisión cfr. Benítez-Burraco, 2005c). Por otro lado, y en relación con la función que se ha 
propuesto para el área de Broca, en particular, en determinadas especies de homínidos (cf. Wilkins y Wakefield, 1995 y la discusión anterior), conviene no olvidar tampoco que la relevancia de esta región para el procesamiento lingüístico ha sufrido últimamente una importante reevaluación, de forma que actualmente se considera que podría constituir simplemente uno de los componentes de la memoria de trabajo verbal (encargada, quizás, de tareas fonológicas) (Desmond y Fiez, 1998; Kaan y Stowe, 2002) y no el área cortical responsable (en exclusividad) del procesamiento sintáctico (a lo sumo se ocuparía, junto con las regiones anejas, como el opérculo, la ínsula y la materia blanca subyacente, de la computación durante la recepción de procesos sintácticos altamente específicos, en particular, de la relación que existe entre los constituyentes sintagmáticos desplazados por las reglas transformacionales y las huellas dejadas por los mismos [Grodzinsky, 2000]).

En definitiva, puede afirmarse que hasta el momento no hemos logrado cuantificar con exactitud la magnitud de los cambios estructurales y funcionales que tuvieron lugar durante la adquisición filogenética de la competencia lingüística por parte de nuestra especie. Y en lo que atañe, en concreto, a la relación entre la lateralización y el lenguaje, lo cierto es que esta peculiaridad de la organización del cerebro debería contemplarse simplemente como un requisito necesario, pero no suficiente, para la existencia de éste último.

\section{2. ¿Qué conclusiones cabría extraer de todo lo discutido acerca de la lateralización y el lenguaje en lo que atañe al modo en que ha evolucionado el lenguaje?}

El análisis crítico de las evidencias paleoneurológicas relacionadas con la lateralización estructural y funcional del cerebro parece poner de manifiesto que la aparición en términos evolutivos de las capacidades cognitivas distintivas de nuestra especie (incluyendo el lenguaje) no habría sido una mera cuestión de incremento de la complejidad estructural, sino fundamentalmente de la creación de un programa idiosincrásico que ha permitido elegir una trayectoria de desarrollo ligeramente diferente a las ya experimentadas previamente por nuestros antecesores y a las existentes actualmente en otras especies de primates. Este hipótesis vendría corroborada por los resultados derivados del análisis de las diferencias que existen a nivel genético entre nuestra especie y las restantes especies de primates superiores, que conciernen fundamentalmente al nivel de expresión de los genes y no tanto a su secuencia, lo cual es particularmente cierto en lo que se refiere a los genes involucrados en la organización y el funcionamiento del cerebro (Enard et al., 2002b; Cáceres et al., 2003; Dorus et al., 2004; Preuss et al., 2004; Uddin et al., 2004; Varki, 2004).

Esta significativa estabilidad filogenética del proteoma cerebral humano (esto es, del conjunto de todas las proteínas implicadas en un determinado proceso, en términos cuantitativos y cualitativos) parece sugerir que el "órgano del lenguaje" sería en buena medida el resultado del reciclado de elementos (moleculares, ontogenéticos, citológicos, histológicos) ya presentes en un momento dado en nuestro organismo como resultado de su historia evolutiva, y no tanto una estructura biológica creada desde cero. Así, y expresado de forma sintética, la modificación de la secuencia de unos pocos genes reguladores (como es el caso de FOXP2) y/o de su perfil transcripcional (como habría sucedido con los genes 
PCDHX [PCDH11X]/PCDHY [PCDH11Y]) habría permitido que, en un breve lapso de tiempo en términos evolutivos, hubiese tenido una lugar una remodelación notable de determinados circuitos neuronales (o la captación de los mismos) y su dedicación (seguramente de forma no exclusiva) a la computación (recursiva) de elementos simbólicos (casos paradigmáticos serían, en la línea de lo discutido en la cuestión anterior, los de las áreas de Broca y Wernicke), cuyos resultados serían transformados por sistemas motores y perceptivos ya existentes (en términos evolutivos) en estructuras exteriorizables e interiorizables. Como quiera que el ajuste final de estos circuitos depende, en atención a la propia dinámica de organización del sistema nervioso central (tal como se caracterizó en la primera de las cuestiones), de los estímulos externos recibidos por el individuo y del contexto ontogenético que suponen las restantes estructuras nerviosas que los rodean y con las que necesariamente interactúan, resulta plausible afirmar que el "órgano del lenguaje" surgido de este proceso evolutivo (i) se hallaría delimitado en términos funcionales ( $\mathrm{y}$, hasta cierto punto, anatómicos); (ii) tendría un carácter indudablemente innato, aunque sólo en parte podría considerarse codificado genéticamente; (iii) en lo que concierne específicamente a la información genética necesaria para su desarrollo que habría que considerar novedosa en términos filogenéticos, lo cierto es que sería muy reducida, puesto que la facultad del lenguaje habría surgido en buena medida de la puesta en contacto de los sistemas encargados del pensamiento y de la percepción, y de la motricidad, que serían los que responderían a programas genéticos más complejos y con una historia evolutiva mucho más dilatada (Hauser et al., 2002); y (iv) se habría visto sometido posteriormente a un proceso de selección "por modificación y descenso" de neto sabor darwiniano, al poseer evidentes ventajas adaptativas (Lieberman, 2002). Es, en definitiva, en este contexto en el que cobra su genuina importancia (y, al mismo tiempo, el que ésta queda debidamente acotada) la cuestión de la lateralización estructural y funcional del cerebro en relación con el origen del lenguaje discutida en este artículo.

\section{Referencias bibliográficas}

Anderson, S. R. y D. W. Lightfoot (2000): “The human language faculty as an organ”. Annu. Rev. Physiol., 62, págs. 697-722.

Arbib, M. A. (2003): "The Evolving Mirror System: A Neural Basis for Language Readiness". En Christiansen, M. H. y S. Kirby (dirs.): Language Evolution. Oxford: Oxford University Press, págs. 182-200.

Arsuaga, J. L. et al. (2000): Claves de la evolución humana: http://www.pntic.mec.es/mem/claves evolucion/guia/guia.html (23-11-2003).

Benítez-Burraco, A. (2003): “Evidencias fósiles del origen del lenguaje?”, Interlingüística, 14, págs. $129-140$

Benítez-Burraco, A. (2005a): "FOXP2: del trastorno específico a la biología molecular del lenguaje. I. Aspectos etiológicos, neuroanatómicos, neurofisiológicos y moleculares”, Rev. Neurol., 40, págs. 671-682.

Benítez-Burraco, A. (2005b): "FOXP2: del trastorno específico a la biología molecular del lenguaje. II. Implicaciones para la ontogenia y la filogenia del lenguaje”, Rev. Neurol., 41, págs. 37-44. 
Benítez-Burraco, A. (2005c): "Caracterización neuroanatómica y neurofisiológica del lenguaje humano", RSEL, 35, págs. 461-494.

Benítez-Burraco, A. (2007): "Genes y lenguaje”, Teorema, 26, págs. 37-71.

Ben-Shachar, M., Hendler, T., Kahn, I., Ben-Bashat, D. y Y. Grodzinsky (2003): "The neural reality of syntactic transformations: evidence from functional magnetic resonance imaging", Psychol. Sci., 14, págs. 433-440.

Benson, D. L., Colman, D. R. y G. W. Huntley (2001): "Molecules, maps and synapse specificity", Nat. Rev. Neurosci., 2, págs. 899-909.

Bishop, D. V. M. (2001): "Individual differences in handedness and specific speech and language impairment: evidence against a genetic link", Behav. Genet., 31, págs. 339-351.

Blanco, P., Sargent, C. A., Boucher, C. A., Mitchell, M. y N. A. Affara (2000): "Conservation of PCDHX in mammals: expression of human $\mathrm{X} / \mathrm{Y}$ genes predominantly in the brain", Mamm. Genet., 11, págs. 906-914.

Botha, R. P. (2002): “Did language evolve like he vertebrate eye?”, Lang. Commun., 22, págs. 131158.

Bradshaw, J. L. y N. C. Nettleton (1981): “The nature of hemispheric specialization in man”, Behav. Brain Sci., 4, págs. 51-63.

Brownell, H. H., Pincus, D., Blum, A., Rehak, A. y E. Winner (1997): "The effects of righthemisphere brain damage on patients' use of terms of personal reference”, Brain Lang., 52, págs. 60-79.

Bruses, J. L. (2000): "Cadherin-mediated adhesion at the interneuronal synapse", Curr. Opin. Cell Biol., 12, págs. 593-597.

Buxhoeveden, D. P., Switala, A. E., Litaker, M., Roy, E. y M. F. Casanova (2001): "Lateralization of minicolumns in human planum temporale is absent in nonhuman primate cortex", Brain Behav. Evol., 57, págs. 349-358.

Cáceres M., Lachuer, J., Zapala, M. A., Redmond, J. C., Kudo, L., Geschwind, D. H., Lockhart, D. J., Preuss, T. M. y C. Barlow (2003): "Elevated gene expression levels distinguish human from nonhuman primate brains", Proc. Nat. Acad. Sci. U.S.A., 100, págs. 13030-13035.

Cantalupo, C. y W. D. Hopkins (2001): “Asymmetric Broca’s area in great apes”, Nature, 414, págs. 505.

Cantalupo, C., Pilcher, D. L. y W. D. Hopkins (2003): "Are planum temporale and sylvian fissure asymmetries directly related? A MRI study in great apes", Neuropsychologia, 41, págs. 19751981.

Corballis, M. C. (2002): From hand to Mouth. Princeton: Princeton University Press.

Corballis, M. C., Lee, K., McManus, I. C. y T. J. Crow (1996): "Location of the handedness gene on the X and Y chromosomes", Am. J. Med. Genet. (Neuropsychiatric Genet.), 67, págs. 50-52.

Coren, S. y C. Porac (1977): "Fifty centuries of right-handedness: the historic record", Science, 198, págs. 631-632.

Crow, T. J. (2001): "ProtocadherinXY: a candidate gene for cerebral asymmetry and language". En Wray, A. (dir.): The Transition to Language. Oxford: Oxford University Press, págs. 93-112.

Damasio, H., Grabowski, T. J., Tranel, D., Hichwa, R. D. y A. R. Damasio (1996): "A neural basis for lexical retrieval", Nature, 300, págs. 499-505.

Dapretto, M., Davies, M. S., Pfeifer, J. H., Scott, A. A., Sigman, M., Bookheimer, S. Y. y M. Iacoboni (2006): "Understanding emotions in others: mirror neuron dysfunction in children with autism spectrum disorders", Nat. Neurosci., 9, págs. 28-30.

Dehaene-Lambertz, G., Dehaene, S. y L. Hertz-Pannier (2002): "Functional neuroimaging of speech perception in infants", Science, 298, págs. 2013-2015. 
Desmond, J. E. y J. A. Fiez (1998): "Neuroimaging studies of the cerebellum: language, learning and memory", Trends Cogn. Sci., 2, págs. 355-362.

Dingwall, W. D. (1995): "Complex behaviors: evolution and the brain”, Behav. Brain Sci., 18, págs. 186-188.

Donald, M. (1995): "Neurolinguistic models and fossil reconstructions", Behav. Brain Sci., 18, págs. 188-189.

Dorus, S., Vallender, E. J., Evans, P. D., Anderson, J. R., Gilbert, S. L., Mahowald, M., Wyckoff, G. J., Malcom, C. M. y B. T. Lahn (2004): "Accelerated evolution of nervous system genes in the origin of Homo sapiens", Cell, 119, págs. 1027-1040.

Enard, W., Przeworski, M., Fisher, S. E., Lai, C. S. L., Wiebe, V., Kitano, T., Monaco, A. P. y S. Pääbo (2002a): "Molecular evolution of FOXP2, a gene involved in speech and language", Nature, 418, págs. 869-872.

Enard, W., Khaitovich, P., Klose, J., Zöllner, S., Heissig, F., Giavalisco, P., Nieselt-Struwe, K., Muchmore, E., Varki, A., Ravid, R., Doxiadis, G. M., Bontrop, R. E. y S. Pääbo (2002b): "Intra- and interspecific variation in primate gene expression patterns", Science, 296, págs. 340-343.

Francks, C., Fisher, S. E., MacPhie, I. L., Richardson, A. J., Marlow, A. J., Stein, J. F. y A. P. Monaco (2002): "A genomewide linkage screen for relative hand skill in sibling pairs", Am. J. Hum. Genet., 70, págs. 800-805. Erratum: Am. J. Med. Genet., 70, págs. 1074.

Francks, C., DeLisi, L. E., Fisher, S. E., Laval, S. H., Rue, J. E., Stein, J. F. y A. P. Monaco (2003): "Confirmatory evidence for linkage of relative hand skill to 2p12-q11", Am. J. Hum. Genet., 72, págs. 499-502.

Frank, M. y R. Kemler (2002): "Protocadherins”, Curr. Opin. Cell Biol., 14, págs. 557-562.

Freedman, M. L. y R. C. Martin (2001): "Dissociable components of short-term memory and their relation to long-term learning", Cogn. Neuropsychol., 18, págs. 193-226.

Friederici, A. D. (2002): "Towards a neural basis of auditory sentence processing", Trends Cogn. Sci., 6, págs. 78-84.

Friederici, A. D. (2006): "The neural basis of language development and its impairment", Neuron, 52, págs. 941-952.

Gannon, P. J., Holloway, R. L., Broadfield, D. C. y A. R. Braun (1998): “Asymmetry of chimpanzee planum temporale: humanlike pattern of Wernicke's brain language area homolog”, Science, 279, págs. 220-222.

Gebbia, M., Ferrero, G. B., Pilia, G., Bassi, M. T., Aylsworth, A. S., Penman-Splitt, M., Bird, L. M., Bamforth, J. S., Burn, J., Schlessinger, D., Nelson, D. L. y B. Casey (1997): "X-linked situs abnormalities result from mutations in ZIC3", Nature Genet., 17, págs. 305-308.

Geschwind, D. H., Miller, B. L., DeCarli, C. y D. Carmelli (2002): "Heritability of lobar brain volumes in twins supports genetic models of cerebral laterality and handedness", Proc. Nat. Acad. Sci. U.S.A., 99, págs. 3176-3181.

Gopnik, M. y M. B. Crago (1991): "Familial aggregation of a developmental language disorder", Cognition, 39, págs. $1-50$.

Gould, S. J. (1991): "Exaptation: a crucial tools for evolutionary psychology”, J. Soc. Issues, 47, págs. 43-65.

Grodzinsky, Y. (2000): “The neurology of syntax: Language use without Broca's area”, Behav. Brain. Sci., 23, págs. 1-71.

Hauser, M. D. y K. Andersson (1994): "Left hemisphere dominance for processing vocalizations in adult, but not infant, rhesus monkeys: field experiments", Proc. Natl. Acad. Sci. U.S.A., 91, págs. 3946-3948.

Hauser, M. D., Chomsky, N. y W. T. Fitch (2002): "The faculty of language: what is it, who has it, and how did it evolve?", Science, 298, págs. 1569-1579. 
Hernández-Muela, S., Mulas, F. y L. Mattos (2004): "Plasticidad neuronal funcional”, Rev. Neurol. 38 (Supl. 1): S58-S68.

Holloway R. L. (1983): "Human paleontological evidence relevant to language behavior", Human Neurobiology, 2, págs. 105-114.

Just, M. A., Carpenter, P. A., Keller, T. A., Eddy, W. F., y B. Thulborn (1996): "Brain activation modulated by sentence comprehension”, Science, 274, págs. 114-116.

Kaan, E. y Stowe, L. A. (2002): "Storage and computation in the brain: a neuroimaging perspective". En Nooteboom, S., Weerman, F. y F. Wijnen (dirs): Storage and Computation in the Language Faculty. Dordrecht: Kluwer, págs. 257-298.

Klar, A. J. S. (1996): “A single locus, RGHT, specifies preference for hand utilization in humans", Cold Spring Harbor Symp. Quant. Biol., 61, págs. 59-65.

Krause, J., Lalueza-Fox, C., Orlando, L., Enard, W., Green, R. E., Burbano, H. A., Hublin, J. J., Hanni, C., Fortea, J., de la Rasilla, M., Bertranpetit, J., Rosas, A. y S. Pääbo (2007): "The Derived FOXP2 Variant of Modern Humans Was Shared with Neandertals”, Curr. Biol., 17, págs. 1908-1912. Lalland, K. N., Kumm J, Van Horn, J. D. y M. W. Feldman (1995): "A gene-culture model of human handedness", Behav. Genet., 25, págs. 433-445.

Leask, S. J. y T. J. Crow (2001): "Word acquisition reflects lateralization of hand skill", Trends Cogn. Sci., 5, págs. 513-516.

Levy, J. y T. Nagylaki (1972): “A model for the genetics of handedness”, Genetics, 72, págs. 117-128. Lieberman, P. (2002): "On the nature and evolution of the neural bases of human language", Am. J. Phys. Anthropol., 45, págs. 36-62.

Liégeois F, Baldeweg T, Connelly A, Gadian DG, Mishkin M. y F. Vargha-Khadem (2003): "Language fMRI abnormalities associated with FOXP2 gene mutation", Nat. Neurosci. 6:1230-1237. Lopes, A. M., Ross, N., Close, J., Dagnall, A., Amorim, A. y T. J. Crow (2006): "Inactivation status of PCDH11X: sexual dimorphisms in gene expression levels in brain”, Hum. Genet., 119, págs. 267-275. Lowe, L. A., Supp, D. M., Sampath, K., Yokoyama, T., Wright, C. V., Potter, S. S., Overbeek, P. y M. R. Kuehn (1996): "Conserved left-right asymmetry of nodal expression and alterations in murine situs inversus", Nature, 381, págs. 158-161.

MacNeilage, P. F. (1998): "The frame/content theory of evolution of speech production", Behav. Brain Sci., 21, págs. 499-511.

Marcus, G. F. y S. E. Fisher (2003): "FOXP2 in focus: what can genes tell us about speech and language?", Trends Cogn. Sci., 7, págs. 257-262.

Martin, R. C. (2003): "Language processing: functional organization and neuroanatomical basis", Annu. Rev. Psychol., 54, págs. 55-89.

McManus, I. C. (1985): "Handedness, language dominance and aphasia: a genetic model", Psychol. Med. Monogr. Supl. 8: S1-S40.

Mummery, C. J., Patterson, K., Price, C. J., Ashburner, J., Frackowiak, R. S. J. y J. R. Hodges (2000): "A voxel-based morphometry study of semantic dementia: relationship between temporal lobe atrophy and semantic memory", Ann. Neurol., 47, págs. 36-45.

Nettle, D. (2003): "Hand laterality and cognitive ability: A multiple regression approach", Brain Cogn., 52, págs. 390-398.

Neville, H. J. y D. Bavelier (1998): "Neural organization and plasticity of language", Curr. Opin. Neurobiol., 8, págs. 254-258.

Olsson, H. y C. Ingvar (1991): "Left handedness is uncommon in breast cancer patients", Europ. J. Cancer, 27, págs. 1694-1695.

Petersen, M. R., Beecher, M. D., Zoloth, S. R., Green, S., Marler, P. R., Moody, D. B. y W. C. Stebbins (1984): "Neural lateralization of vocalizations by Japanese macaques: communicative significance is more important than acoustic structure", Behav. Neurosci., 98, págs. 779-790. 
Pinker, S. (2001): El instinto del lenguaje. Madrid: Alianza Editorial.

Poremba, A., Malloy, M., Saunders, R. C., Carson, R. E., Herscovitch, P. y M. Mishkin (2004): "Species-specific calls evoke asymmetric activity in the monkey's temporal poles", Nature, 427, págs. 448-451.

Preuss, T. M. (2004): "What is it like to be a human? En Gazzaniga, M. S. (dir.): The cognitive neurosciences III. Cambridge: MIT Press, págs. 5-22.

Risch, N. y K. R. Merikangas (1996): "The future of genetic studies of complex human diseases", Science, 273, págs. 1516-1517.

Rizzolatti, G. y M. A. Arbib (1998): "Language within our grasp”, Trends Neurosci., 21, págs. 188194.

Rizzolatti, G., Fadiga, L., Gallese, V. y L. Fogassi (1996): "Premotor cortex and the recognition of motor actions", Brain Res. Cogn. Brain Res., 3, págs. 131-141.

Sargent, C. A., Briggs, H., Chalmers, I. J., Lambson, B., Walker, E. y N. A. Affara (1996): "The sequence organization of $\mathrm{Yp}$ /proximal $\mathrm{Xq}$ homologous regions of the human sex chromosomes is highly conserved”, Genomics, 32, págs. 200-209.

Shapiro, L y D. R. Colman (1999): "The diversity of cadherins and implications for a synaptic adhesive code in the CNS", Neuron, 23, págs. 427-430.

Shriberg, L. D., Ballard, K. J., Tomblin, J. B., Duffy, J. R., Odell, K. H. y C. A. Williams (2006): "Speech, prosody, and voice characteristics of a mother and daughter with a 7,13 translocation affecting FOXP2", J. Speech Lang. Hear. Res.49: 500-525.

St George, M., Kutas, M., Martinez, A. y M. I. Sereno (1999): "Semantic integration in reading: engagement of the right hemisphere during discourse processing", Brain, 122, págs. 1317-1325.

Thomas, C., Altenmilller, E., Marchmann, G., Kahrs, J. y J. Dichgans (1997): "Language processing in aphasia: changes in lateralization patterns during recovery reflect cerebral plasticity in adults", Electroencephalogr. Clin. Neurophysiol., 102, págs. 86-97.

Tobias, P. V. (1983): "Recent advances in the evolution of the hominids with especial reference to brain and speech". En Chagas, C. (dir.): Recent advances in the evolution of primates. Ciudad del Vaticano: Pontificia Academia Scientiarum, págs. 85-140.

Tobias, P. V. (2003): "Orígenes evolutivos de la lengua hablada". En Gutiérrez Lombardo, R., Martínez Contreras, J. y J. L. Vera Cortés (dirs.): Naturaleza y Diversidad Humana. Estudios Evolucionistas. México: Centro de Estudios Filosóficos, Políticos y Sociales "Vicente Lombardo Toledano", págs. 285-307.

Uddin, M., Wildman, D. E., Liu, G., Xu, W., Johnson, R. M., Hof, P. R., Kapatos, G., Grossman, L. I. y M. Goodman (2004): "Sister grouping of chimpanzees and humans as revealed by genome-wide phylogenetic analysis of brain-gene expression profiles", Proc. Nat. Acad. Sci. U.S.A., 101, págs. 2957-2962.

Van Agtmael, T., Forrest, S. M. y R. Williamson (2002): "Parametric and non-parametric linkage analysis of several candidate regions for genes for human handedness", Europ. J. Hum. Genet., 10, págs. 623-630.

Van Lancker, D. R. y D. Kempler (1987): "Comprehension of familiar phrases by left- but not by right-hemisphere-damaged patients”, Brain Lang., 32, págs. 265-277.

Vargha-Khadem, F., Watkins, K. E., Alcock, K. J., Fletcher, P. y R. E. Passingham (1995): "Praxic and nonverbal cognitive deficits in a large family with a genetically transmitted speech and language disorder", Proc. Natl. Acad. Sci. U.S.A., 92, págs. 930-933.

Vargha-Khadem, F., Gadian, D. G., Copp, A. y M. Mishkin (2005): "FOXP2 and the neuroanatomy of speech and language", Nat. Rev. Neurosci., 6, págs. 131-138.

Varki A. (2004): “How to make an ape brain”, Nat. Genet., 36, págs. 1034-1036. 
Watkins, K. E., Dronkers, N. F. y F. Vargha-Khadem (2002): "Behavioural analysis of an inherited speech and language disorder: comparison with acquired aphasia”, Brain, 125, págs. 452-64.

Whitcombe, E. (1995): "Paleoneurology of language: Grounds for scepticism", Behav. Brain Sci., 18, págs. 204-205.

Wilkins, W. K. y J. Wakefield (1995): "Brain evolution and neurolinguistic preconditions", Behav. Brain Sci., 18, págs. 161-182, 205-226.

Wilson, N. D., Ross, L. J. N., Crow, T. J. y E. V. Volpi (2006): "PCDH11 is X/Y homologous in Homo sapiens but not in Gorilla gorilla and Pan troglodytes", Cytogenet. Genome Res., 114, págs. 137-139.

Witelson, S. F. y W. Pallie (1973): "Left hemisphere specialization for language in the newborn: Neuroanatomical evidence for asymmetry”, Brain, 96, págs. 641-646.

Yamagata, K., Andreasson, K. I., Sugiura, H., Maru, E., Dominique, M., Irie, Y., Miki, N., Hayashi, Y., Yoshioka, M., Kaneko, K., Kato, H. y P. F. Worley (1999): "Arcadlin is a neural activity-regulated cadherin involved in long-term potentiation”, J. Biol. Chem., 274, págs. 19473-11979.

Yeo, R. A., Gangestad, S. W., Thoma, R., Shaw, P. y K. Repa (1997): "Developmental instability and cerebral lateralization", Neuropsychology, 11, págs. 552-561.

Zhou, X., Sasaki, H., Lowe, L., Hogan, B. L. M. y M. R. Kuehn (1993): "Nodal is a novel TGF-betalike gene expressed in the mouse node during gastrulation", Nature, 361, págs. 543-547. 